\title{
Studies on Nanoengineered Multilayer Capsules for Drug Delivery
}

Faten Z.Mohamed ${ }^{1}$, Mohamed A. Shemis ${ }^{2}$,Mona N.Mohareb² and Heba A.Hosiny ${ }^{1}$

1 Biochemistry Department, Faculty of science, Zagazig university, Egypt.

2 Biochemistry and Molecular Biology Dep, Theodor Bilharz Research Institute, Giza, Egypt.

\section{ARTICLE INFO}

Keywords:

LbL, polyelectrolyte microcapsules, MTT, MDBK cell line

\begin{abstract}
A B S T R A C T
Background: Polymeric multilayer capsules are now being engineered to encapsulate various classes of drug molecules, by using polymers that are biodegradable or that can respond and release their payload in response to well-defined stimuli and are produced by stepwise adsorption of oppositely charged polymers onto the surface of colloidal particles followed by core dissolution, which is called Layer by Layer technique, Aim: The present study focuses on synthesis and characterization of polyelectrolyte multilayer microcapsules which was loaded with Bovine Serum Albumin and the cytotoxic effect of the microcapsules on the living cells was studied, Materials and methods: The microcapsules were fabricated by using Poly(allylamine) Hydrochloride (PAH) and Poly(styrenesulfonate) (PSS) as the counter charge polyelectrolytes and biocompatible calcium carbonate as the template which is highly promising to produce capsules for biological applications and loaded with bovine serum albumin (BSA) as a model drug, BSA labeled with fluorescein isothiocyanate (BSA-FITC), Calcium carbonate particles and the microcapsuls were characterized by SEM and TEM ,The effect of microcapsules on Madin Darby Bovine Kidney (MDBK) cell line were studied by using The 3-[4,5-dimethylthiazol2-y]-2, 5-diphenyltetrazolium bromide MTT assay, Results: $\mathrm{CaCO}_{3}$ microparticles are very convenient template for the preparation of polyelectrolyte microcapsules $1-4 \mu \mathrm{m}$, preloading method is more effective than post loading method, Our results confirmed that this synthetic $\mathrm{CaCO}_{3}$ microcapsules are cytocompatible, Conclusion: We thus have shown that the very easily prepared, stable, prototypical microcapsules composed of PSS/PAH are useful drug delivery agents for proof-of-principle drug delivery studies.
\end{abstract}

(C) 2013 Publisher All rights reserved.

\section{INTRODUCTION}

Drug-delivery science is driven by the need to develop systems that can deliver precise quantities of a therapeutic payload at a specific target site or tissue at tailored release rate and 
a specific trigger ${ }^{[1,2]}$. This technique is of special interest for medicine, particularly for treatment of cancer diseases which demands rather toxic and expensive medical substances [3]. Several drug molecules cannot be formulated or administered by conventional techniques as they exhibit poor water solubility or suffer from limited stability in a complex environment such as the human body. A beautiful example of a novel system that has recently emerged from cross-disciplinary scientific symbiosis is polymeric multilayer capsules (PMLCs) ${ }^{[4] .}$

PMLCs have been extensively explored for their physicochemical properties since their advent in the late 1990s ${ }^{[5]}$, and more recently they have attracted attention for drugdelivery applications ${ }^{[6]}$. Polyelectrolyte microcapsules are produced by stepwise adsorption of oppositely charged polymers onto the surface of colloidal particles followed by core dissolution, which is called (Layer by Layer) technique ${ }^{[7,8]}$. Using this approach, a variety of materials, including charged and uncharged species, have been successfully assembled . Commercially available synthetic polyelectrolytes such as PSS, PAH, PDADMAC, Particularly, some naturally occurring polyelectrolytes, such as polysaccharides are eyecatching ${ }^{[9]}$. The sacrificing of the encapsulated template is a key step for capsule fabrication. Several templates have been used for the capsule preparation, such as weakly cross-linked melamine-formaldehyde lattices, silica particles, polystyrene lattices and biological templates ${ }^{[10,11,12]}$. However, the dissolution of many polymer cores such as melamineformaldehyde and polystyrene requires strong acids or organic solvents, and in some cases template materials cannot be completely removed from the capsules because of the interaction between templates and capsule walls, Among these cores only carbonate particles can be decomposed under rather mild conditions ${ }^{[13]}$, By varying the intermixing speed, time, $\mathrm{pH}$ value and ratio of initial ingredients during precipitation such $\mathrm{CaCO}_{3}$ templates are produced ${ }^{[14]}$. Some proteins, such as bovine serum albumin and peroxidase, were incorporated into hollow polyelectrolyte capsules by regulating permeability of the multilayer shell for macromolecules ${ }^{[15]}$.

To evaluate the potential of PMLCs for drug delivery it is important to understand their interactions with living cells. One of the most important parameters is toxicity. Several research groups have assessed this topic by performing in vitro cell-viability assays such as the MTT test ${ }^{[16,17]}$. The present study focuses on the microcapsules based on Poly(allylamine) (PAH) and Poly(styrenesulfonate) (PSS) as the counter charge polyelectrolytes and biocompatible calcium carbonate as the template which is highly promising to produce capsules for biological applications, as it shows low cytotoxic and can be easily removed with EDTA. Bovin serum albumin (BSA) was chosen as the model protein [18]

In this study we have focused on loading of BSA-FITC into the microcapsules by preloading and post loading method, studying the effect of $\mathrm{PH}$ on the synthetic microcapsules for loading and release of bovine serum albumin, characterization of the capsules by optical microscope and electron microscope and the cytotoxic effect of this microcapsules on Madin Darby Bovine Kidney (MDBK) cell line by The 3-[4,5-dimethylthiazol-2-y]-2, 5diphenyltetrazolium bromide MTT assay. Good cytocompatibility of microcapsules consist of synthetic polyelectrolytes was confirmed. Their application as drug microlnanotransporters can be envisaged and will be the subject of future investigations.

\section{MATERIALS AND METHODS:}

\section{(1)Materials:}

PSS ,PAH, EDTA 99+\%, MTT isomer I and fluorescein isothiocyanate (FITC) calcium chloride dehydrate $99,99 \%$, sodium carbonate $99,99 \%$ were purchased from SigmaAldrich ; Sodium chloride 99,5\% from Biostain ; Bovine serum albumin $>98.0 \%$ From BIO BASIC INC; Sodium carbonate buffer(0.1M, PH 9), MEM Earle's medium with LGlutamine, HEPES, Antibiotics PEN-STREP contain 10,000U pencillin/ml ,10,000 $\mu \mathrm{g}$ 
Streptomycin/ml and $25 \mu \mathrm{g}$ Amphotericin B/ml were purchased from Lonza- swiss company ; 5\% heat-inactivated fetal bovine serum, L-Glutamine, DUlbecco's Phosphate Buffered Saline (PBS) without magnesium and calcium, Trypsin-ethylenediaminetetraacetic acid 1X in PBS were purchased from Biowest. All chemicals of high quality and purity. MDBK cells were provided by tissue culture laboratory of VACSERA were prepared from a stock stored in liquid nitrogen, at Theodore bilharz research institute.

\section{(2) Methods:}

\section{Fabrication of polyelectrolyte microcapsules:}

Polyelectrolyte microcapsules were prepared using $\mathrm{CaCO}_{3}$ micro-particles as a sacrificial template. $\mathrm{CaCO}_{3}$ micro-particles were synthesized according to Volodkin et al ${ }^{\text {[19] }}$ by mixing $\mathrm{CaCl}_{2}$ and $\mathrm{Na}_{2} \mathrm{CO}_{3}$ solutions $(0.33 \mathrm{M})$ with vigorous stirring for $30 \mathrm{~s}$ followed by extensive washing with pure water to remove unreacted reagents. Spherically shaped $\mathrm{CaCO}_{3}$ micro-particles with an average diameter of 1 to $4 \mu \mathrm{m}$ were obtained. For synthesis of $\mathrm{CaCO}_{3}(\mathrm{PSS})$ particles Firstly, PSS $(4 \mathrm{~g} / \mathrm{L})$ was mixed $\mathrm{CaCl}_{2}(0.33 \mathrm{M})$ under magnetic agitation $(1000 \mathrm{rpm})$, Then an equal molar of $\mathrm{Na}_{2} \mathrm{CO}_{3}$ solutions was rapidly poured into a flask together with $\mathrm{CaCl}_{2}$ solution contained PSS. The $\mathrm{CaCO}_{3}$ particles were coated using the layer-by-layer technique by dispersing them in PSS solution $(2 \mathrm{mg} / \mathrm{ml} 0.5 \mathrm{M} \mathrm{NaCl})$. After 10 min shaking the micro-particles were collected by centrifugation, and residual PSS was removed by washing twice with pure water. Thereafter the micro-particles were suspended in PAH solution $(2 \mathrm{mg} / \mathrm{ml} 0.5 \mathrm{M} \mathrm{NaCl})$ and shaked for $10 \mathrm{~min}$, followed by centrifugation and two washing steps. This procedure was repeated until the desired number of layers was deposited (five bilayers in this study). Hollow capsules were obtained by removing the $\mathrm{CaCO}_{3}$ core by incubating the coated micro-particles for $10 \mathrm{~min}$ in $1 \mathrm{ml}$ of $0.2 \mathrm{M}$ EDTA solution ( $\mathrm{pH} 5.5$ ) to dissolve the $\mathrm{CaCO}_{3}$. The dissolved ions were then removed by three centrifugation and washings steps. Finally the capsules were re-suspended in $1 \mathrm{ml}$ PBS. The capsule concentration was determined by haemocytometry.

\section{Labeling of Bovine serum albumin with Fluorescein isothiocyanate (FITC):}

BSA was labeled with FITC for loading it into the micro-cpsules by preloading and post loading method and detecting its loading by fluorescence microscope. BSA was labeled with FITC by Amicon Ultra-15 Centrifugal Filters (5KDa) according to ${ }^{[20]} .5 \mathrm{mg} / \mathrm{ml}$ of BSA that was dissolved in $(0.1 \mathrm{M}$ sodium carbonate buffer, $\mathrm{pH} 9)$ was mixed with FITC solution (3 $\mathrm{mg}$ in $1 \mathrm{ml} 0.1 \mathrm{M}$ sodium carbonate buffer $\mathrm{PH} 9$ ) then was shaked orbital overnight at room temperature protected from light. after the end of incubation of BSA-FITC were moved into $50 \mathrm{ml}$ amicon ultra-15 (5KDa) filters and the volume was completed with deionized water to $15 \mathrm{ml}$ and centrifuged at $3500 \mathrm{rpm}$ for $25 \mathrm{~min}$ then the downstream are trashed and upstream was completed with water and repeat till get clear downstream which when checked on UV are not flure anymore. The upstream should be yellow in color then the absorbance of BSAFITC was measured at $280 \mathrm{~nm}$ for BSA and $495 \mathrm{~nm}$ for FITC. Then Calculate protein concentration according to the Beer-Lambert law as follows:

$$
\text { Protein concentration }(M)=\frac{A_{280}-\left(A_{\max } \times C F\right)}{\varepsilon_{\text {protein }}} \times \text { dilution factor }
$$

- sprotein= protein molar extinction coefficient (e.g., the molar extinction coefficient of BSA is $\sim 43824 \mathrm{M}^{-1} \mathrm{~cm}^{-1}$ )

$$
\text { - } C F=\text { Correction factor }=\frac{A_{280}}{A_{\max }}=0.3
$$




\section{Determination of Encapsulation Efficiency of BSA into the microcapsules}

Preloading method: Effectiveness of protein entrapping (immo-bilization) was determined by a difference between optical density of the initial solution and a supernatant obtained after protein adsorption into the micro-particles ${ }^{[21]}$. $\mathrm{CaCO}_{3}$ particles with BSA -FITC adsorbed on

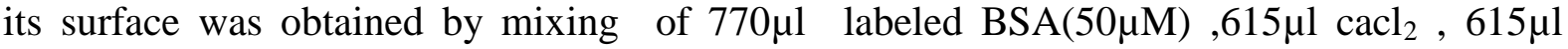
$\mathrm{Na}_{2} \mathrm{CO}_{3}$ then centrifuged then measure absorbance of BSA and FITC supernatant using UVVIS spectrophotometer then the amount of BSA adsorbed on the particles was detected by subtracting the concentration of BSA in supernatant from original concentration $(50 \mu \mathrm{M})$.

Encapsulation efficiency $=($ concentration of encapsulated protein $/$ total protein concentration ) $\times 100$

Post loading method: the effect of Temperature, $\mathrm{pH}$ on the loading of BSA -FITC into the microcapsules was studied.

\section{A-Temperature effect:}

A solution of $70 \mathrm{kDa}$ BSA that labeled with FITC in a concentration of $50 \mu \mathrm{M}$ was added to the microcapsules and was left one to two hours for incubation at room temperature. During this time the mixed solution was wrapped in aluminum foil in order to protect it from light. In the meanwhile a water bath was heated to $68{ }^{\circ} \mathrm{C}$ in which the tubes were placed subsequently for the heating. They were left for 30min in thermal treatment for the shrinking process protected from light. Afterwards the suspensions were allowed to cool down to room temperature for $5 \mathrm{~min}$ and washed twice to remove the non-encapsulated FITC-BSA. Then absorbance of BSA, FITC was measured to determine the encapsulation efficiency.

\section{B-pH effect :}

The effect of $\mathrm{pH}$ on BSA- FITC loading was studied. The $\mathrm{pH}$ of BSA- FITC studied was in the range of 3.4 to 5.0. After mixing $2 \times 10^{8}$ microcapsules with $200 \mu \mathrm{L}$ ( $1 \mathrm{mg} / \mathrm{mL}$ BSAFITC) , the mixture was incubated for 12 hours at ambient temperature, followed by centrifugation at $3500 \mathrm{rpm}$ for 5 minutes. FITC,BSA was quantified, using a UV-vis spectrophotometer, according to its absorbance peak at $280,495 \mathrm{~nm}^{[22]}$. The post loading of BSA- FITC was also confirmed using a fluorescence microscope.

\section{Determination of the amount of Released BSA from the microcapsules:}

The BSA- FITC concentration was measured using spectrophotometer. The amount of unloaded protein and overall loss during capsules washing was deducted from the initial amount of BSA- FITC added to the system to give the total amount of encapsulated BSAFITC. To investigate the release of BSA from the capsules, the BSA- FITC -loaded microcapsules were mixed with $1 \mathrm{~mL}$ phosphate-buffered saline (PBS; $\mathrm{pH} 7.4$ ) or $1 \mathrm{~mL} \mathrm{HCl}$ solution (pH 5.0 or 1.0) and incubated at ambient temperature under gentle shaking. A 200$\mu \mathrm{L}$ aliquot of supernatant was taken out at a given time and supplemented with $200 \mu \mathrm{L}$ fresh PBS or $\mathrm{HCl}$ solution. Each $200 \mu$ Lof supernatant was diluted with PBS or $\mathrm{HCl}$ solution, the absorbance of FITC, BSA was recorded, and the cumulative release of FITC-BSA was calculated. The results were analyzed by excel program.

\section{Characterization of polyelectrolyte microcapsules:}

Optical Microscopy: The zeiss model microscope, equipped with 10x, 40x and 100x lenses and a Coolsnap Color camera were used for bright field inspection and recording ${ }^{[23]}$.

Electrophoretic mobility. The electrophoretic mobility of the polyelectrolyte microcapsules was measured in deionized water at room temperature using a Malvern Zetasizer 2000 (Malvern, UK). The zeta-potential ( $\zeta$-potential) was calculated from the electrophoretic mobility $(\mu)$ using the Smoluchowski function $\zeta=\mu^{*} \eta / \varepsilon$ where $\eta$ and $\varepsilon$ are the viscosity and permittivity of the solvent, respectively. 
Scanning Electron Microscopy: A drop of capsule suspension was placed on a silicon wafer (a metal bead) and dried under a nitrogen stream, and then coated with gold. SEM images were recorded with LEO 1550 VP scanning electron microscope (SEM, Inspect S, FEI company, JAPAN) operated at $5 \mathrm{kV}$.

Transmission Electron Microscopy: The hollow and filled structures of the microcapsules were observed by transmission electron microscopy (TEM) (EM 208 S Philips, Japan). Nickel grids sputtered with carbon films were used to support the microcapsules. Ten microlitre of the microcapsule suspension was put on the Nickel grids and air-dried before measurement.

Fluorescence microscope: The FITC labelled microcapsules were imaged with fluorescence microscope.

\section{Viability assay:}

MTT assays were used to measure the viability of MDBK cells cultured in 2D in the presence of microcapsules ${ }^{[24]}$. The cells were cultured in MEM Earle's medium containing $10 \%$ fetal bovine serum, 1\% antibiotic ( penicillin-streptomycin- Amphotericin B 100x), 1\% L-glutamine, $1 \%$ HEPES in a humidified incubator at $37{ }^{\circ} \mathrm{C}$, with $5 \% \mathrm{CO} 2$ and $95 \%$ relative humidity. For determination of cytotoxicity of the microcapsules on MDBK cells , 100 $\mu 1$ of cell suspension at density of $10^{4}$ cells was added to plate wells and were incubated for $12 \mathrm{~h}$ to allow cells to attach onto the surface. Then microcapsules were added serially at $1 \times 10^{8}, 1 \times 10^{7}, 1 \times 10^{6}, 1 \times 105,1 \times 10^{4}, 1 \times 10^{3} / \mathrm{ml}$, the microcapsules was diluted using MEM Earle's medium . Four samples (in four wells on the same plate) were prepared at each microcapsule concentration. Four control wells with cells $\left(10^{4}\right.$ but no microcapsules were also prepared. well of only media was prepared for background measurement and two well for blank solvent, also wells contain only low and high concentration of microcapsules The plate was placed in an incubator at $37{ }^{\circ} \mathrm{C}$ for $24 \mathrm{~h}$. Then $20 \mu \mathrm{l}$ of MTT reagent was added to each well, Incubate the plate for approximately $2-4$ hours at $37^{\circ} \mathrm{C}$ in $\mathrm{CO}_{2}$ incubator. The plate was examined periodically for the appearance of punctate, intracellular precipitate (formazane crystals) using an inverted microscope. When purple precipitate is clearly visible under the microscope the culture medium was aspirated from each well carefully to prevent disruption or the precipitate. The resulting MTT formazane crystals were dissolved with $100 \mu 1$ acidified isopropanol solution, The plates were ready within 1 hour after adding acidified isopropanol solution.

After the end of incubation time, pipetting up and down was required to completely dissolve the MTT formazane crystals. Absorbance at wavelength of $570 \mathrm{~nm}$ was spectrophotometrically measured. Cell viability is expressed as

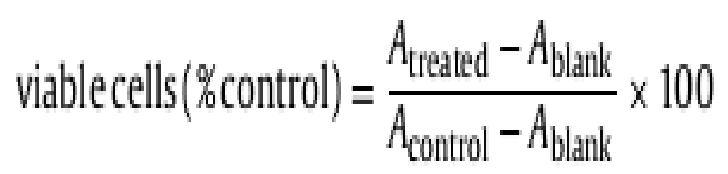

Where $\mathrm{A}_{\text {treated }}$ is the average absorbance in wells containing cells cultured with a particular concentration of microcapsules, $\mathrm{A}_{\text {blank }}$ is the absorbance of the MTT solvent, and $\mathrm{A}_{\text {control }}$ is the average absorbance in wells containing cells but no microcapsules. The results of MTT assay were analyzed by excel program. 


\section{RESULTS:}

Encapsulation efficiency of BSA-FITC into the microcapsules:

For the preloading method: protein concentration was calculated according to the BeerLambert law

Encapsulation Efficiency = (concentration of encapsulated protein / total concentration $) \times$ $100=(43.1 \div 50) \times 100=86 \%$

For postloading method: In the case of temperature effect: the encapsulated amount of protein is $7 \mu \mathrm{M}$ due to The capsules gradually shrink with increasing temperature The shrinkage process is mainly powered by decreasing the area of the water/polyelectrolyte interface. Encapsulation Efficiency $=14 \%$

In the case of pH effect: Encapsulation Efficiency $=78.8 \%$

Our results showed high encapsulation efficiency in the case of preloading method due to the high porosity of calcium carbonate particles, followed by $\mathrm{pH}$ effect due to any changes in $\mathrm{pH}$ effect on the charge balance of polyelectrolye layers of the microcapsules and permeability to the proteins with high molecular weight (BSA) but in the case of temperature effect, by increasing the temperature, the microcapsules was shrinked and the wall thickness of the polyelectrolytes increased that lead to permeability to the proteins with high molecular weight decreased as shown in Figure (1) .

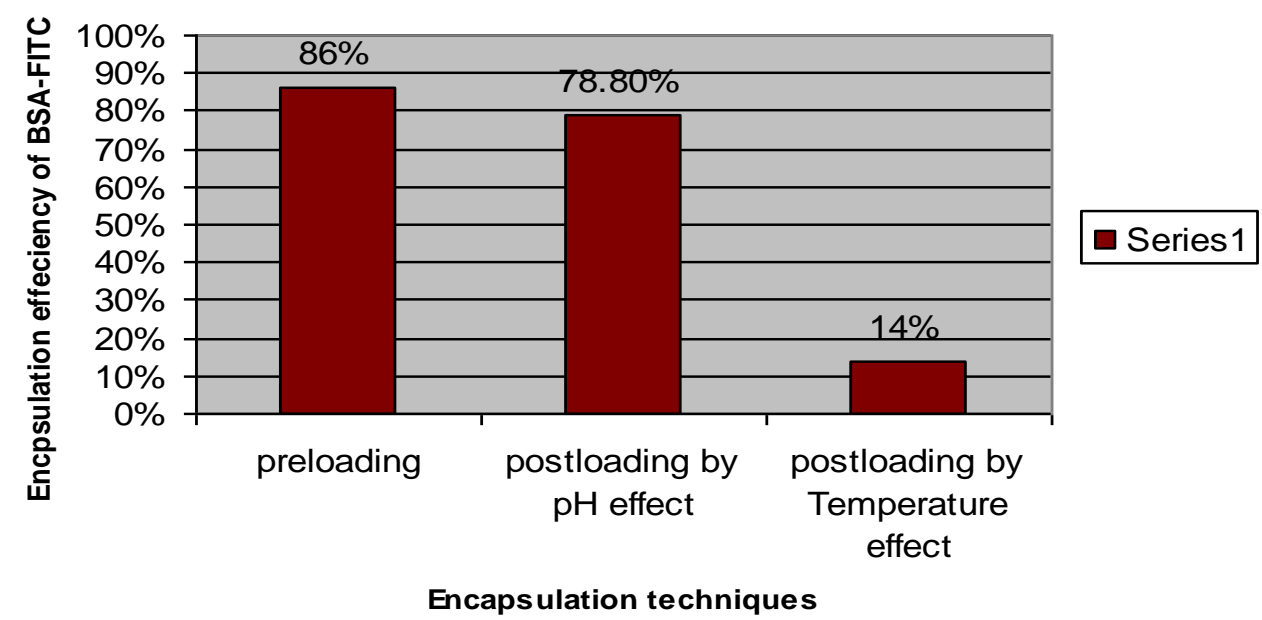

Figure (1): Encapsulation efficiency of BSA-FITC into the microcapsules by different techniques .

\section{Cumulative release of BSA-FITC from PMLCs under different pH values:}

After loading of BSA-FITC into the microcapsules at PH 3.8, $39.4 \mu \mathrm{M}$ was encapsulated, the results show that the amount of protein released in the case of phosphate-buffered saline (PBS; $\mathrm{pH} 7.4$ ) more than $\mathrm{HCl}$ solution ( $\mathrm{pH} 5.0$ or 1.0) as in (figure 2). 
Table 1. Cumulative release of BSA-FITC from PMLCs under different $\mathrm{pH}$ values.

\begin{tabular}{|l|l|l|l|l|}
\hline PH & $\begin{array}{l}\text { Initial } \\
\text { concentration } \\
\text { of BSA-FITC }\end{array}$ & $\begin{array}{l}\text { Concentration of } \\
\text { encapsulated } \\
\text { protein }\end{array}$ & $\begin{array}{l}\text { Amount of } \\
\text { protein } \\
\text { released } \\
\text { after 30min }\end{array}$ & $\begin{array}{l}\text { Amount of } \\
\text { protein } \\
\text { released after } \\
\text { 90min }\end{array}$ \\
\hline PBS (7.4) & $50 \mu \mathrm{M}$ & $39.4 \mu \mathrm{M}$ & $44 \%$ & $77 \%$ \\
\hline HCL(5) & $50 \mu \mathrm{M}$ & $39.4 \mu \mathrm{M}$ & $23 \%$ & $56 \%$ \\
\hline HCL(1) & $50 \mu \mathrm{M}$ & $39.4 \mu \mathrm{M}$ & $14 \%$ & $33 \%$ \\
\hline
\end{tabular}

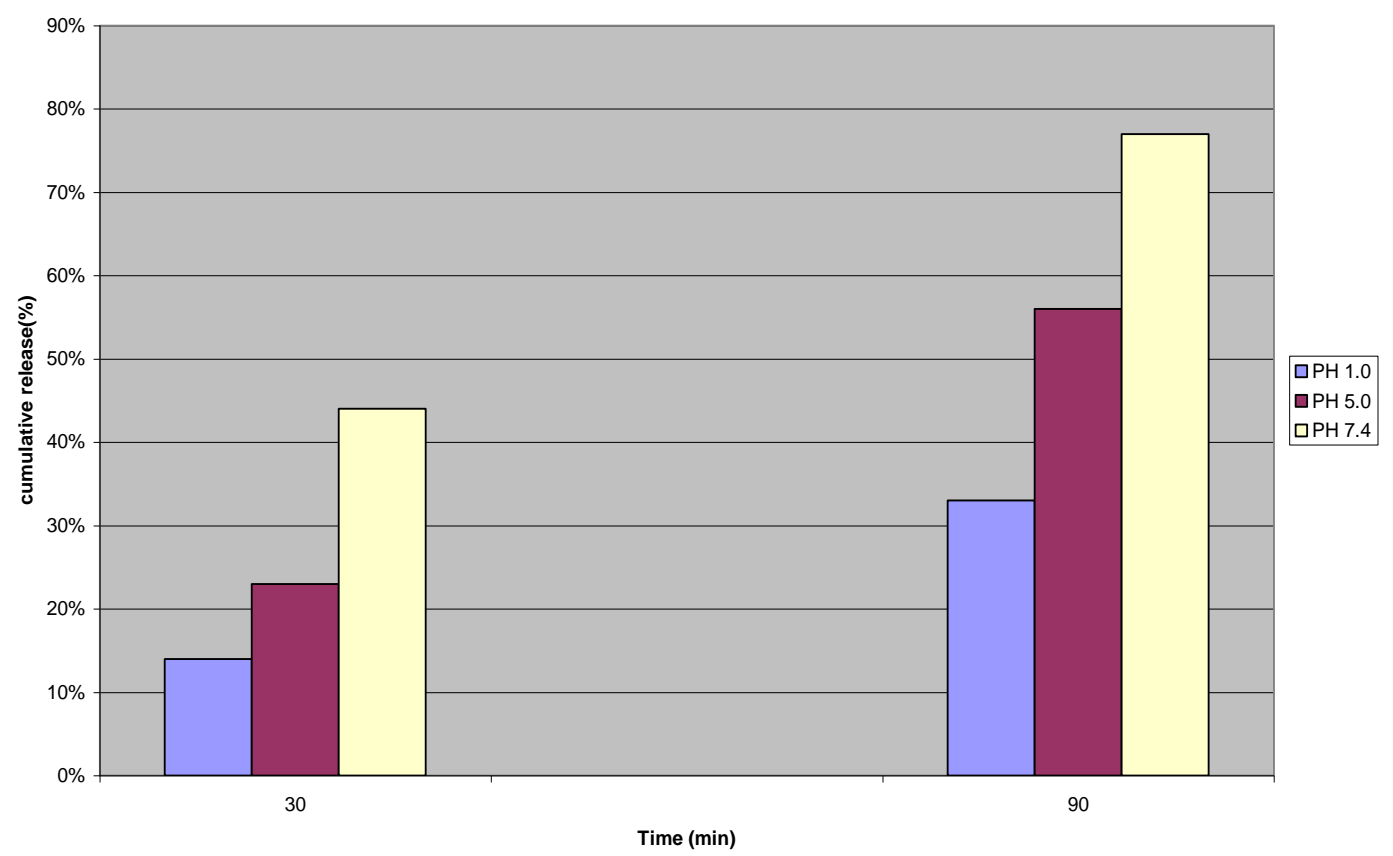

Figure (2): FITC-BSA cumulative release from microcapsules containing PSS versus incubation time at 30 and 90 minutes. Release was significantly different between the $\mathrm{pH}$ values tested. Release was conducted at $37^{\circ} \mathrm{C}$ at $\mathrm{pH} 7.4,5.0$, and 1.0 solutions. 


\section{Characterization of the prepared Polyelectrolyte micro-capsules:}

Electrophoretic mobility of $\mathrm{CaCO}_{3}$ micro-particles and microcapsules: Zeta analyzer measurements show that $\mathrm{CaCO}_{3}$ micro-particles containing BSA adsorbed on it had negative charge $-21.7 \mathrm{mV}$ and the microcapsules with (PAH/PSS)5 exhibited a value of $-25.7 \mathrm{mV}$ as shown in (figure 3 ) .

(A)

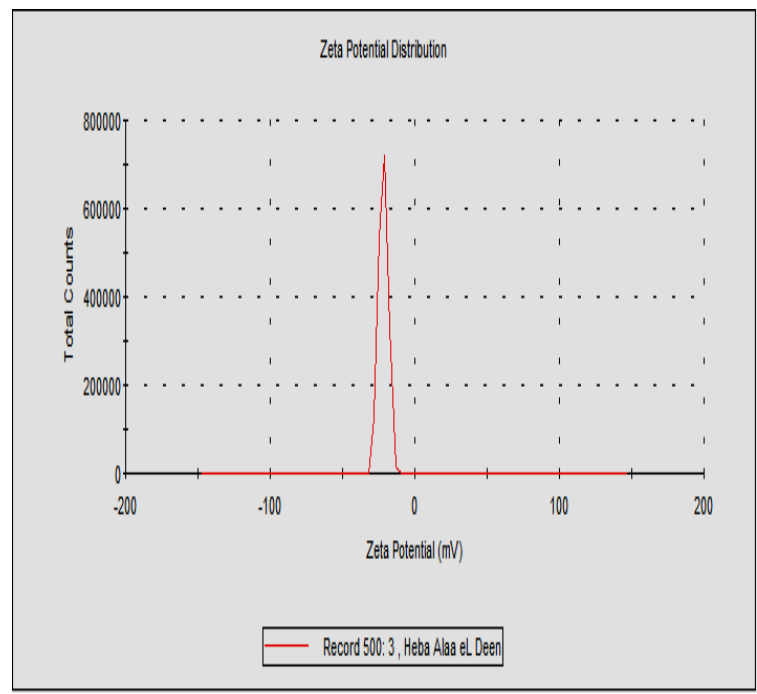

(B)

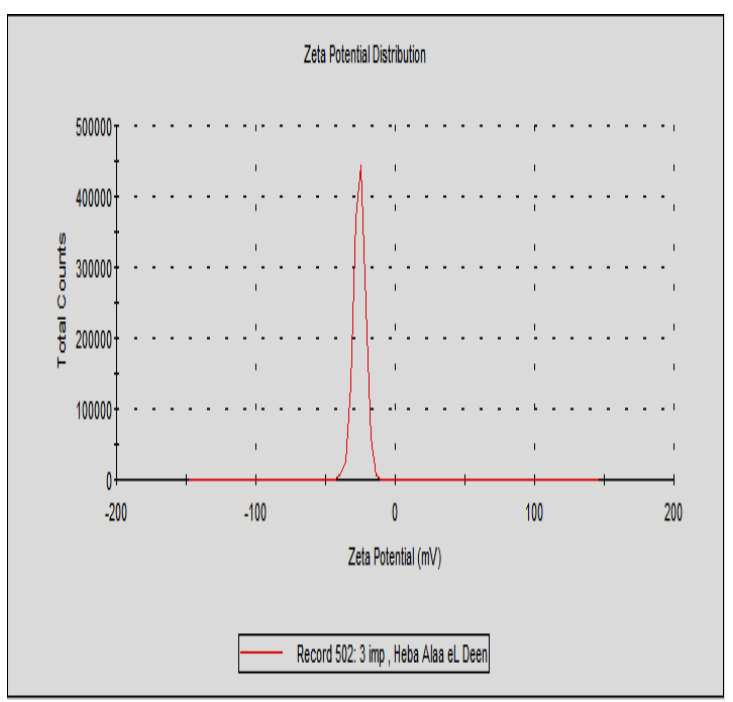

Figure (3) :(A) Zeta potential of $\mathrm{Caco}_{3}$ microparticles containing PSS, (B) Zeta potential of microcapsules with (PAH/PSS)

Optical microscope : Light microscopy was utilized to demonstrate the external shape of microcapsules, the micro photo that were taken show microcapsules are spherical ,monodiperse and non-aggregated as sown in (figure 4).

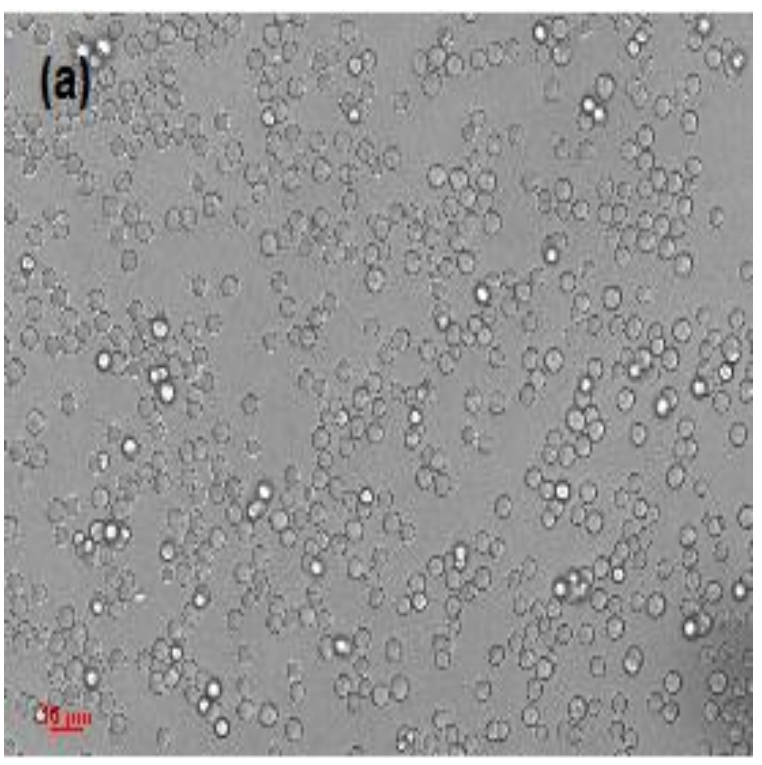

Figure(4): Photos of $\mathrm{CaCO} 3$ micro-particles (a) and the micro-particle-based polyelectrolyte(PSS/PAH) 5 (b). optical microscopy, $100 \mathrm{X}$ magnification.

\section{(b)}

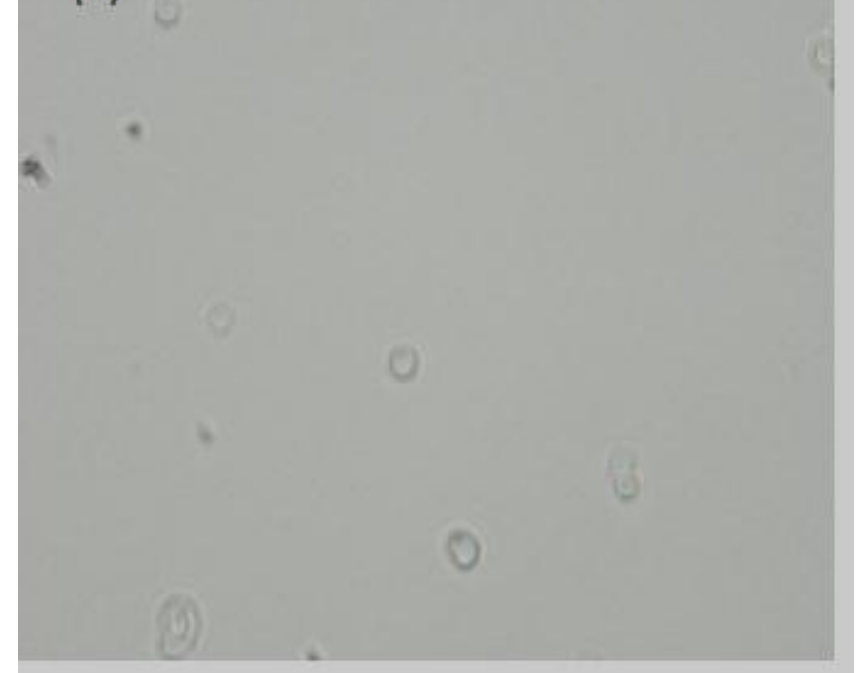


Scanning Electron Microscopy: SEM was utilized to demonstrate the morphology difference between $\mathrm{CaCO}_{3}$ core and microcapsules, size of them and precipitation of $\mathrm{BSA}$ on the capsules as shown in (figure5).
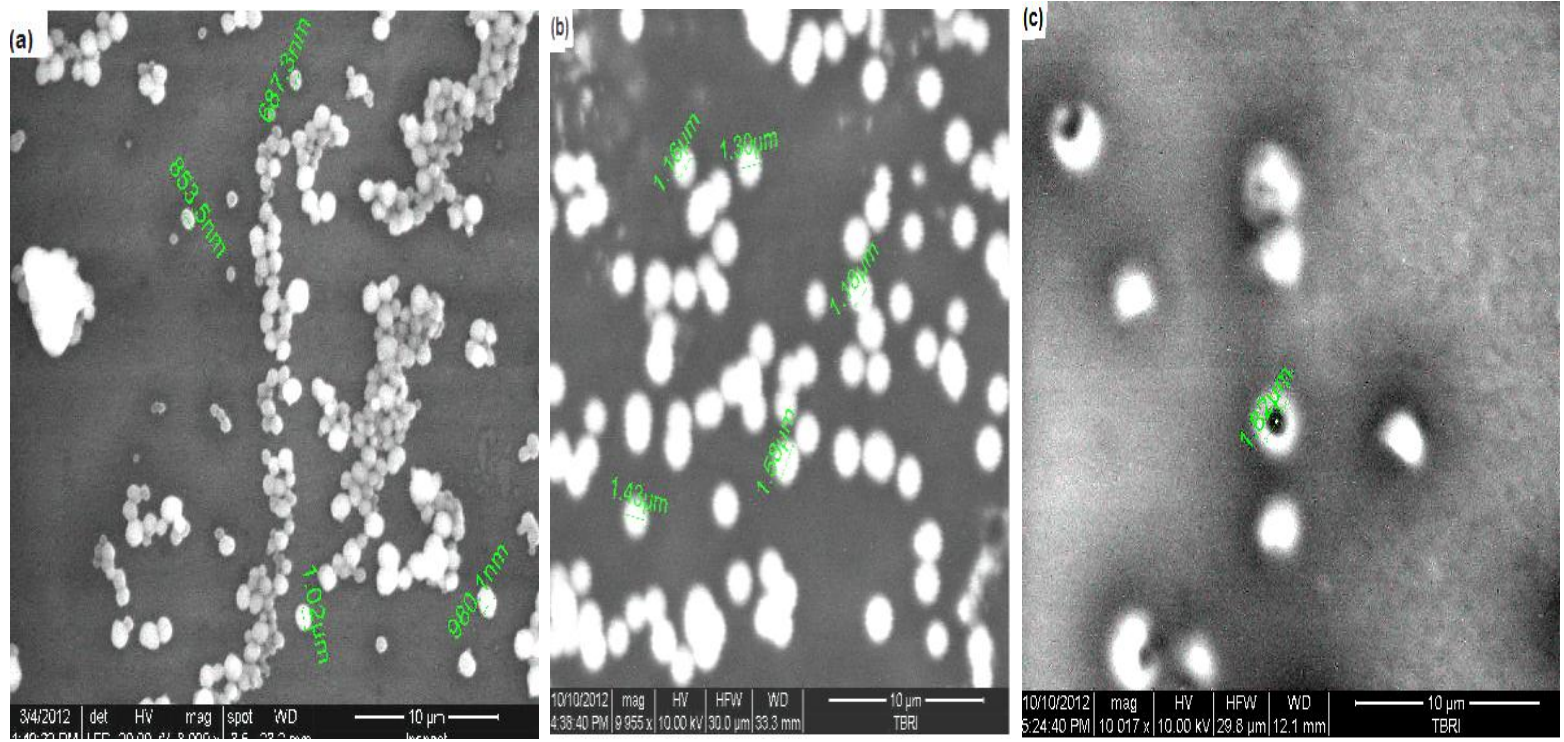

Figure. 5 . photos of $\mathrm{CaCO}_{3}$ micro-particles (a) aggregated with small size, (b) nonaggregated and $\mathrm{CaCO}_{3}$ microcapsules (c) obtained by scanning electron microscope. those differences(shape and size) between a and $b$ are due to the different conditions used during the synthesis of the micro-particles including stirring speed and room temperature.

Transmition electron microscopy: TEM was utilized to demonstrate the precipitation of BSA within the microcapsules, The morphology changes between cores and the microcapsules as shown in (figure 6).
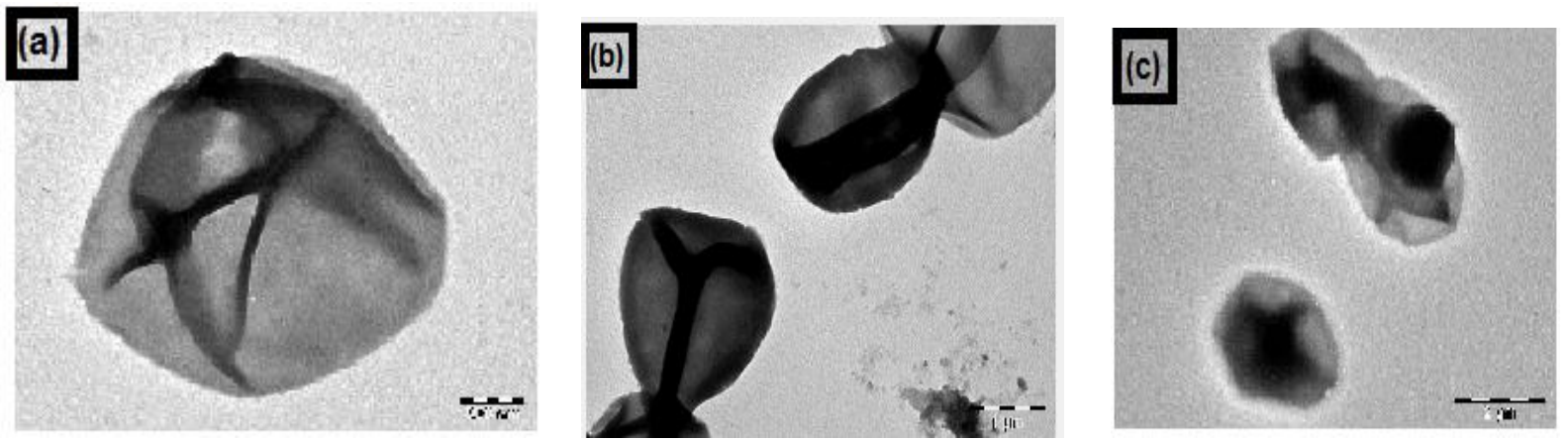

Figure (6): TEM images of $\mathrm{CaCO}_{3}$ microcapsules with collapsed layers (A) The hollow capsules are collapsed completely due to the absence of templated cores and the evaporation of water $(B, C)$ The dark shadows in the capsule centers undoubtedly depict the existence of BSA that deposited between the polelectrolyte layers after dissolution of $\mathrm{CaCO}_{3}$ particles . 
Fluorescence microscopy: was used to determine the loading of BSA-FITC in to the $\mathrm{CaCO}_{3}$ particle and microcapsules.

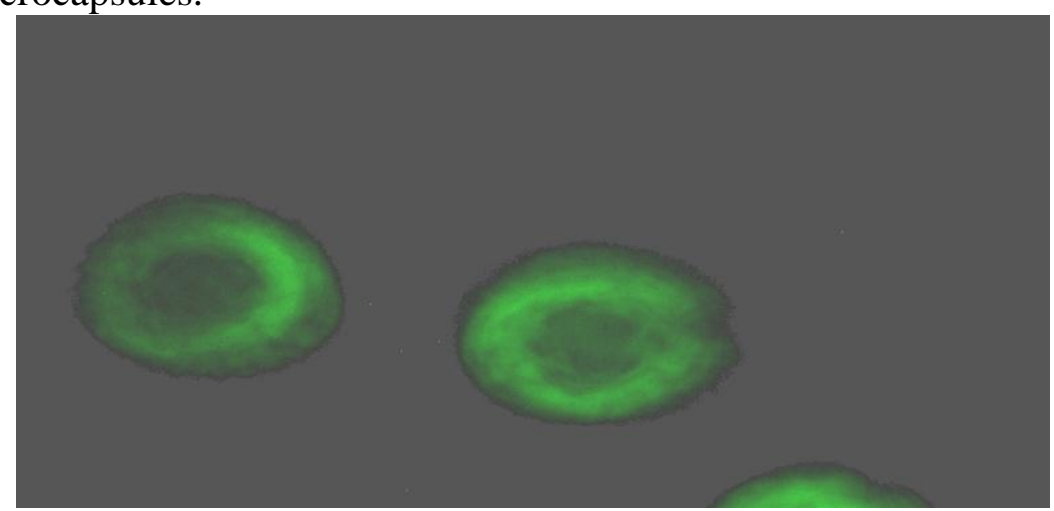

Figure (7): Image of $\mathrm{CaCO}_{3}$ micro-particles by fluorescence microscope after incubation with FITC- BSA.

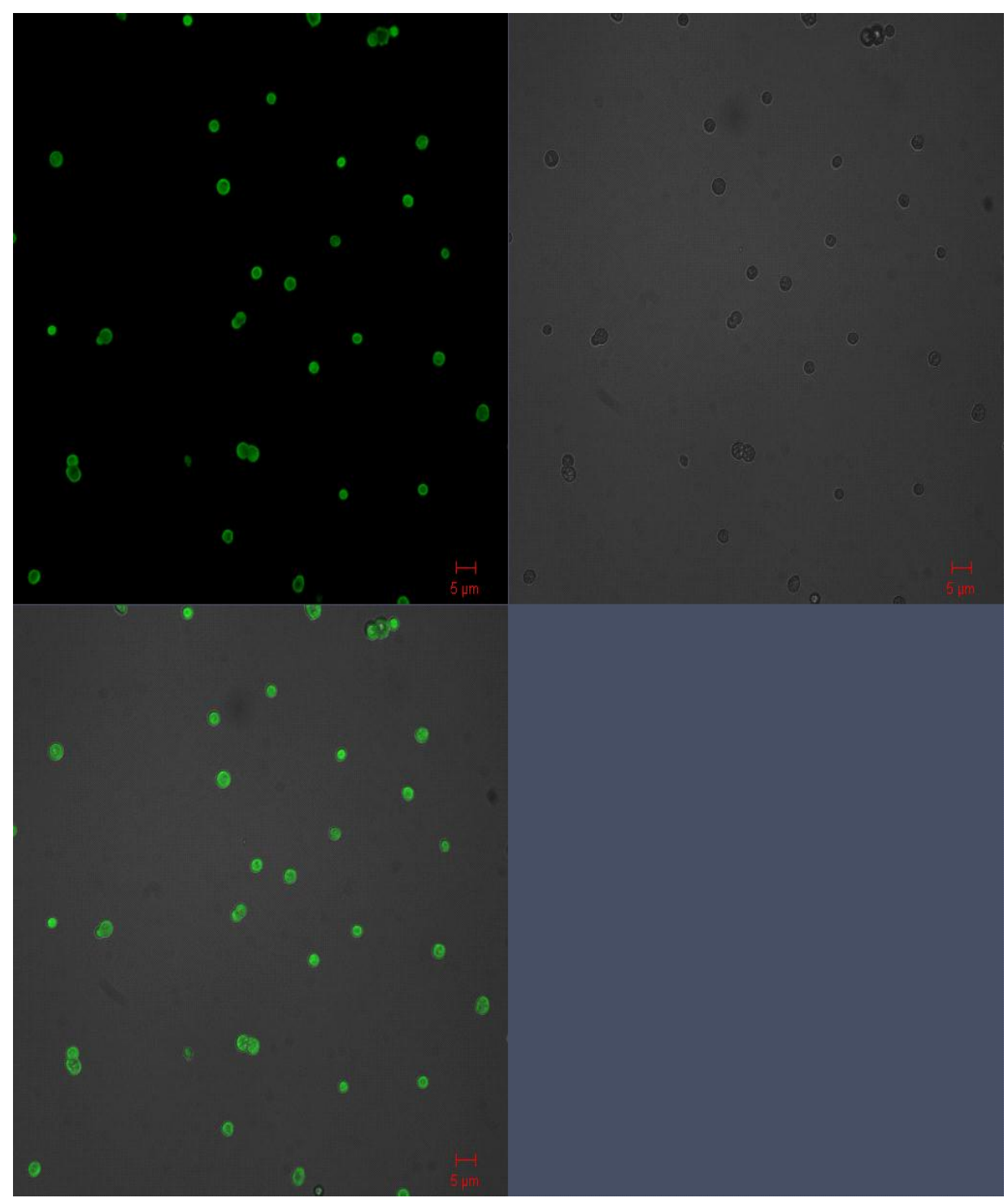

Figure (8): fluorescence images typical $\mathrm{CaCO}_{3}$ capsules with encapsulated $\mathrm{BSA}$, green color in the image is due BSA which was fluorescently labelled with FITC adsorbed on cores. 


\section{Effect of the synthetic microcapsules on cell viability:}

The effects of the PSS/PAH microcapsules and PAH/PSS microcapsules on the viability of MDBK cell line were examined by the MTT assay While PSS/PAH microcapsules are inexpensive and straight forward to prepare as well as exceptionally stable, (Figure. 9a) shows normal MDBK cell line, (figure. 9b) shows image of $105 / \mathrm{ml}$ cells incubated with $10^{7} / \mathrm{ml} 1$ $4 \mu \mathrm{m}$ microcapsules, indicate little decrease in cell viability after incubation with the microcapsules $(1-4 \mu \mathrm{M})$, the present results indicate that MDBK cells cultured with $1-4 \mu \mathrm{m}$ PAH/PSS microcapsules have viability percentage ranging between $83 \%$ to $94 \%$ while cells

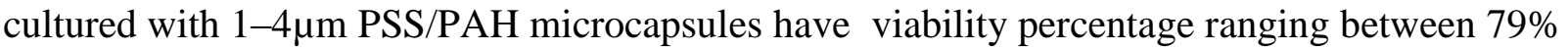
to $92 \%$ as shown in (figure 9c). With increasing the microcapsules concentration, cell viability decreased due to decreasing the conversion of MTT in to the formazane crystals by mitochondrial reductase enzyme producing by viable cells.

(a)

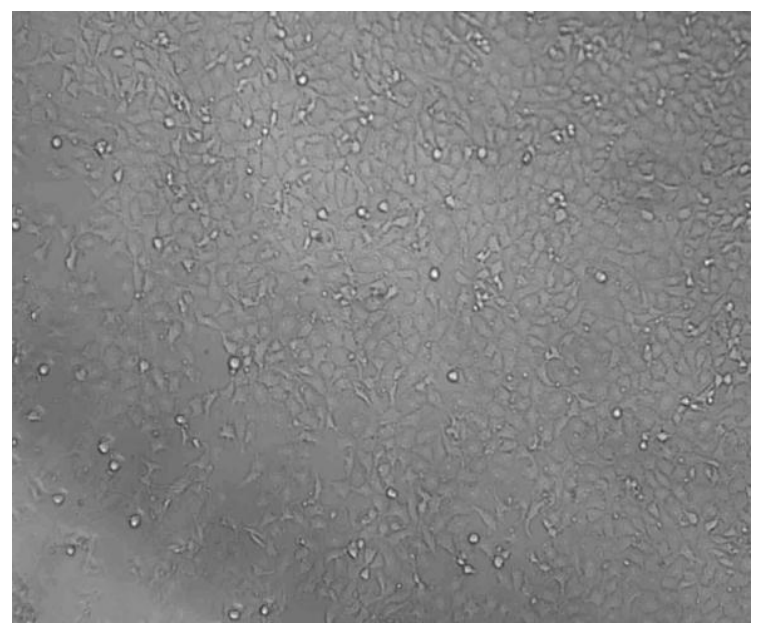

(b)

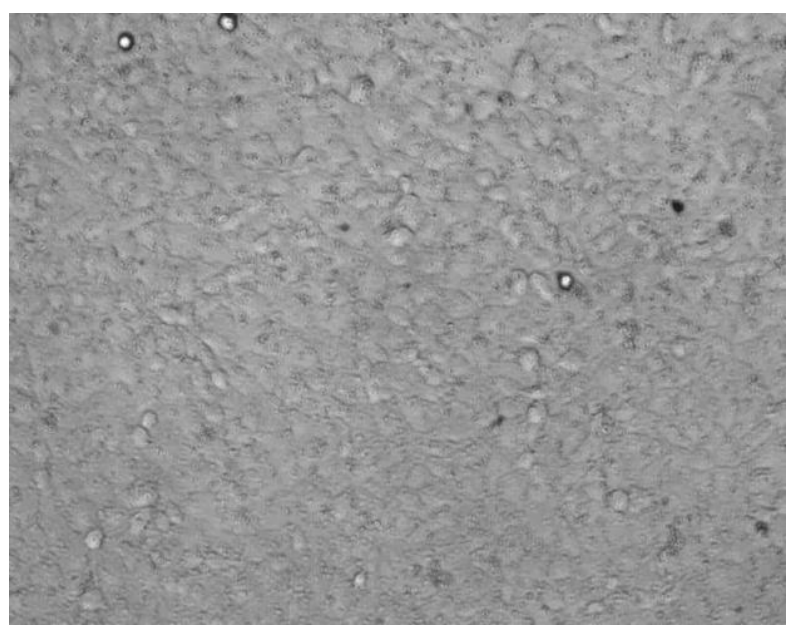

(c)

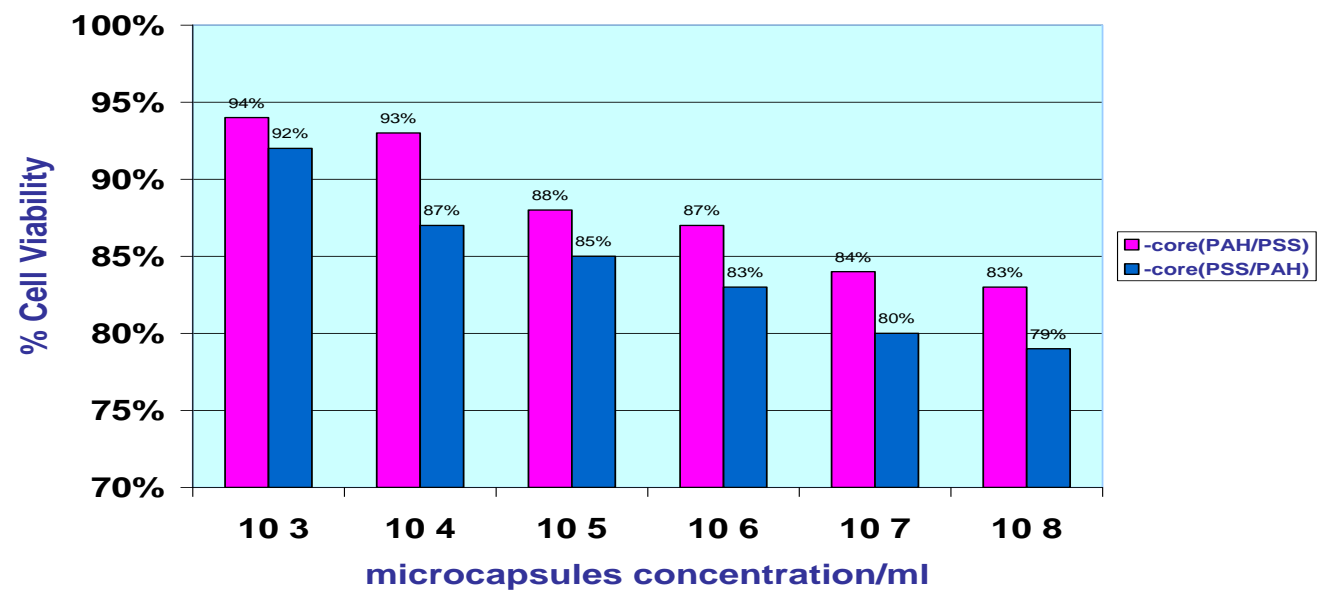

Figure (9): (a) show MDBK cell sheet after incubation $24 \mathrm{~h}$ with MEM Earle's medium, (b) MDBK cell line incubated with concentration 108 of the microcapsules by inverted microscope (c) Number of viable cells relative to cells cultured in the absence of microcapsules as measured by MTT assay as a function of microcapsules with different concentration . 


\section{DISCUSSION:}

$\mathrm{CaCO}_{3}$ microparticles were used as templates for the synthesis of hollow microcapsules using the LbL technique ${ }^{[25]}$, The quality of the resultant microparticles was found to be strongly dependent on the experimental conditions such as type of salts used, their concentration, $\mathrm{pH}$ values of $\left(\mathrm{Cacl}_{2}, \mathrm{Na}_{2} \mathrm{CO}_{3}, \mathrm{PSS}, \mathrm{PAH}\right)$, room temperature, rate of solution mixing and intensity agitation of the reaction mixture ${ }^{[26,27]}$. These microcapsules were obtained by first adsorbing positively and negatively charged polyelectrolytes namely the PSS/PAH pair onto a $\mathrm{CaCO}_{3}$ core. After coating, the $\mathrm{CaCO}_{3}$ cores were dissolved in EDTA solution. EDTA sequested $\mathrm{Ca}^{2+}$ ions, leading to the production of hollow micro-capsules made of five bilayered (PSS/PAH) loaded with BSA with diameter similar to that of the starting $\mathrm{CaCO}_{3}$ cores $(1-4 \mu \mathrm{m})$ as shown in (figure $\left.4 \mathrm{a}, \mathrm{b}\right)$. BSA as protein model was loaded into $\mathrm{CaCO}_{3}$ particles during mixing $\mathrm{cacl}_{2}$ and $\mathrm{Na}_{2} \mathrm{CO}_{3}$ solutions by coprecepitation method (preloading method) ${ }^{[28]}$, The $\mathrm{CaCO}_{3}$ microparticles obtained by this simple route are uniform and homogeneously sized, non-aggregated and highly porous spheres (Figure 4 a).

Our results are in agreement with Volodkin et al who reported that more than $80 \%$ of just-prepared porous $\mathrm{CaCO}_{3}$ microparticles without dispersants recrystallized after storage overnight in water ${ }^{[29]}$. PSS possess functional groups on its long hydrophilic chains , which can complex with $\mathrm{Ca}^{2+}$ and disperse them to avoid agglomerating when calcium carbonate particles growing, In the presence of PSS, the polydispersity of the calcium carbonate particles was decreased and more homogeneous calcium carbonate particles with the size around $4 \mu \mathrm{m}$ were obtained ${ }^{[30]}$.

During crystallization and formation of $\mathrm{CaCO}_{3}$ particles , BSA-FITC $(50 \mu \mathrm{M})$ solution was added to salt mixing solutions. The resultant particles were spherical. One observed capture and spontaneous accumulation of the labeled macromolecules within the particle interior as shown in (figure 7) and by calculating the concentration of non-adsorbed BSA(6.9 $\mu \mathrm{M})$ in the supernatant, we determined the concentration of Adsorbed BSA on the particles which equal to $43.1 \mu \mathrm{M}$, This result mean high encapsulation efficiency of BSA-FITC in the preloading method as shown in (figure 1). Our results are in line with Volodkin et al who observed that a significantly large adsorption of protein on the surface of $\mathrm{CaCO}_{3}$ particles even when both species possess the same sign of charge. Under PH effect, concentration of post loaded protein is $39.4 \mu \mathrm{M}$, this result main that changing $\mathrm{PH}$ effect on charge balance of the polyelectrolytes and causing disassembly of capsules layers leading to high loading of protein in to the capsules. Our results are in line with Zhao and $\mathrm{Li}$ who found that .,more FITC-BSA was loaded into the microcapsules at a lower $\mathrm{pH}^{[31]}$ and Petrov et al who found that polyelectrolyte capsules composed of weak polyelectrolytes are responsive to the $\mathrm{pH}$ of the environment ${ }^{[32]}$.

The release rate of BSA was higher at a higher $\mathrm{pH} .77 \%$ of BSA was released at 90 min at $\mathrm{pH} 7.4$, and by contrast, only $56 \%$ and $33 \%$ of BSA were released at $\mathrm{pH} 5.0$ and 1.0, respectively. The release (e.g., at 30 and $90 \mathrm{~min}$ ) was significantly different between $\mathrm{pH}$ values 7.4, 5.0, and 1.0 (Figure 2). Our results are in line with many authors, Sui and Schlenoff who found that multilayers composed of weak polyelectrolytes can be affected and eventually destroyed at extreme $\mathrm{pH}$ conditions. This phenomenon is due to the $\mathrm{pH}$-induced imbalance of charges, and they ultimately lead to the permeability change of the capsule walls ${ }^{[33]}$, Tian et al., who found that the synergetic controlled release of BSA-FITC can be triggered very well by bio-stimulus - DNA nuclease recognition capsule decomposition, as well as $\mathrm{pH}$ value and salt concentration ${ }^{[34]}$. Zeta analyzer measurements showed that $\mathrm{CaCO}_{3}$ microparticles containing BSA adsorbed on it had negative charge $-21.7 \mathrm{mV}$ and the microcapsules with (PAH/PSS) 5 exhibited a value of $-25.7 \mathrm{mV}$ as shown in (figure 3 ).

SEM was utilized to demonstrate the morphology difference between $\mathrm{CaCO}_{3}$ core and microcapsules, size of them and precipitation of BSA on the capsules, (Figure 5) shows 
microphotos of $\mathrm{CaCO}_{3}$ micro-particles (a) aggregated with small size, (b) non-aggregated obtained, those differences between $\mathrm{a}$ and $\mathrm{b}$ are due to the different conditions used during the synthesis of the micro-particles including stirring speed and room temperature and (Figure 5c) shows microphotos of $\mathrm{CaCO}_{3}$ microcapsules obtained by scanning electron microscope. TEM was utilized to demonstrate the precipitation of BSA within the microcapsules, The hollow nature of capsules is revealed in Figs. 6a. ${ }^{[35]}$.

The dark shadows in the capsule centers undoubtedly depict the existence of BSA as shown in figure $6(\mathrm{~b}, \mathrm{c})$. Figure 8 presents typical $\mathrm{CaCO}_{3}$ capsules with encapsulated BSAFITC, our results are in accordance with stein et al., who found that in the case of $\mathrm{CaCO}_{3}$ capsules the most of encapsulated material is condensed on inner side of the capsule matrix wall, meaning that BSA is not homogeneously distributed in the capsule interior ${ }^{[36]}$.

MTT assay was used for determination of the effect of polyelctrolyte microcapsules that end with PSS or PAH on viability of MDBK cell line, MTT system measures the activity of living cells via mitochondrial dehydrogenase activity . Several studies have determined the effect of the PMLCS on different types of cell lines but MDBK cell line was not used before. The viability was determined from the equation for the different concentration of microcapsules, Figure 9a show MDBK cell line after incubation $24 \mathrm{~h}$ with MEM Earle's medium, figure 9b indicate little decrease in cell viability after incubation with microcapsules, figure 9c shows cell viability as a function of different concentration for 1-4 $\mu \mathrm{m}$ PSS/PAH microcapsules and PAH/PSS microcapsules. With increasing the microcapsules concentration, cell viability decreased due to decreasing the conversion of MTT in to the formazane crystals by mitochondrial reductase enzyme producing by viable cells, this mean that capsules ended with PSS as outermost layer have less effect on the viability of this cells due to PSS is negatively charged so that the capsules remain on the top of cell line and decrease in viability occur due to mechanical stress for competition with cells for surface area but in the case of PSS/PAH capsules viability decrease due to PAH is positively charged and penetrate inside the cells and caused decreasing in cell proliferation.

Our results do not agree with De Koker et al., who found that PSS/PAH microcapsules are inexpensive and straight forward to prepare as well as exceptionally stable, their use in invitro studies has been limited by concerns about cytotoxicity, particularly due to the presence of the high concentration of sulfonic ${ }^{[37]}$, While we are in accordance with many authors including Tryoen-Toth et al., who found that PSS did not affect cell health when used as the outermost layer of a planar multilayer film, plating cells on a film of a particular material may not induce the same effects as culturing cells with microcapsules fabricated of identical material ${ }^{[38] \text {. }}$

\section{CONCLUSIONS:}

It could be concluded that the very easily prepared, stable, prototypical microcapsules composed of PSS/PAH are useful drug delivery agents for proof of principle drug delivery studies.

\section{REFERENCES:}

[1] Langer R., Tirrell D. A., (2004) : Designing materials for biology and medicine Nature ; 428: 487-492.

[2] Wang X., Xing F., Zhang M., Han N., Qian Z., (2013): Experimental Study on Cementitious Composites Embedded with Organic Microcapsules Materials; 6: 40644081.

[3] LaVan D. A., McGuire v, Langer R., (2003) : Small-scale systems for in vivo drug delivery. National Biotechnology; Oct ;21(10):1184-1191. 
[4] Antipov A.A., Sukhorukov G.B., (2004) : Polyelectrolyte multilayer capsules as vehicles with tunable permeability . (review) Advances in Colloid \& Interface Science; 111(1-2): 49-61.

[5] Decher G., (1997): Fuzzy nanoassemblies: Toward layered polymeric multicomposites. Science; 277 :1232-1237.

[6] Sukhorukov G.B., Rogach A.L., Garstka M., Springer S., Parak W.J., Munoz-Javier A., Kreft O., Skirtach A.G., Susha A.S., Ramaye Y., Palankar R., Winterhalter M., (2007): Multi-functionalized polymer microcapsules: novel tools for biological and pharmacological applications. Small $3:$ 944-955.

[7] Wang, C., Ye, S., Dai, L., Liu, X., Tong, Z. (2007) :Enhanced resistance of polyelectrolytemultilayer microcapsules to pepsin erosion and release properties of encapsulated indomethacin. Biomacromolecules $8: 1739$.

[8] Li, Z., Lee, D., Rubner, M.F., Cohen, R.E. (2005) : Layer-by-layer assembled janus microcapsules.Macromolecules 38:7876.

[9] Wang, Z.J., Qian, L., Wang, X.L., Yang, F., Yang, X.R. (2008) : Construction of hollowDNA/PLL microcapsule as a dual carrier for controlled delivery of DNA and drug. Colloids Surf. A: Physicochem. Eng. Aspects $326: 29$.

[10] Salaün, F., Vroman, I. (2008) : Influence of core materials on thermal properties of melamine-formaldehyde microcapsules, Eur. Polym. J. 44 : 849.

[11] Mauser, T., Déjugnat, C., Möhwald, H., Sukhorukov, G.B. (2006) : Microcapsules made of weak polyelectrolytes: templating and stimuli-responsive properties. Langmuir. 22: 5888 .

[12] Donath, E., Moya, S., Neu, B., Sukhorukov, G.B., Georgieva, R., Voigt, A., B“aumler, H., Kiesewetter, H., M"ohwald, H. (2002): Hollow polymer shells from biological templates: fabrication and potential applications. Chem. Eur. J. 8: 5481-5485.

[13] Shenoy, D.B., Antipov, A.A., Sukhorukov, G.B., Möhwald, H.(2003): Layer-by-layer engineering of biocompatible, decomposable core-shell structures Biomacromolecules. 4: 265.

[14] Yashchenok A., Parakhonsky B., Donatan S., Kohler D., Skirtach A., Mohwald H., (2013): Polyelectrolyte multilayer microcapsules templated on spherical, elliptical and square calcium carbonate particles J. Mater. Chem B, 1: 1223-1228.

[15] Goodwin A. P., Zhang Y., Ma L., Deng X. and Cheng J., (2013): Triggerresponsive chain-shattering polymers.Polym. Chem; 4:224-228.

[16] De Koker S., Hoogenboom R., De Geest B.G., (2012): Polymeric multilayer capsules in drug delivery.Chemical Society Reviews ; 41: 2867-2884.

[17] An Z.H., Kavanoor K., Choy M.L., Kaufman L.J., (2009): Polyelectrolyte microcapsule interactions with cells in two- and three-dimensional culture. Colloids Surf. B; 70: 114-123.

[18] Yang X.L., Han X., Zhu Y.H., (2005) : (PAH/PSS) $)_{5}$ microcapsules templated on silica core: encapsulation of anticancer drug DOX and controlled release study. Colloids Surface. A: Physicochem. Eng.Aspects;264:49.

[19] Volodkin D. V., Petrov A. I., Larionova N. I., Sukhorukov G. B., (2003): COST 840 \& XI International BRG Workshop on Bioencapsulation. State of Art of Bioencapsulation Science and Technology, Strasbourg (Illkirch), France.

[20] Harlow, E. and Lane, D., Antibodies a Laboratory Manual, pp. 353- 355 (Cold Spring Harbor Laboratory, 1988).

[21] Balabushevich N. G., Tiourina O. P., Volodkin D. V., Larionova N. I., Sukhorukov G.B., (2003): Loading the multilayer dextran sulfate/protamine-microsized-capsules-withperoxidase. Biomacromolecules; 4(5): 1191. 
[22] Gao C., Donath E., Möhwald H., Shen J.C., (2002): Spontaneous deposition of watersoluble substances into microcapsules: phenomenon, mechanism, and application. Angew Chem Int Ed Engl ; 41:3789-93.

[23] Koolman J., and RohmK. H., (1998): Naglyadnaya biokhimiya (Taschenatlas Der Biochemie), Moscow.

[24] Mosmann., (1983): Rapid colorimetric assay for cellular growth and survival: application to proliferation and cytotoxicity assays. J. Immunological Methods; 65 (1-2): 5563.

[25] Decher G., Hong J. D., (1991) : Buildup of ultrathin multilayer films by a selfassembly process.Macromol. Chem., Macromol. Symp; 46: 321-327.

[26] Sukhorukov G.B., Fery A., Brumen M., Möhwald H., (2004): Physical chemistry of encapsulation and release. PCCP; 6: 4078-4089.

[27] Volodkin D.V., Larionova N.I., Sukhorukov G.B., (2004): Protein encapsulation via porous CaCO3microparticles templating. Biomacromolecules; 5 : 1962-1972.

[28] De Geest B.G., Willart M.A., Lambrecht B.N., Vervaet C., Remon J.P., Grooten J., De Koker S., (2012): Surface engineered polyelectrolyte multilayer capsules - synthetic vaccines mimicking microbial structure and function. Angewante Chemie International Edition; 51: 3862-3866.

[29] Volodkin D.V., Petrov A.I., Prevot M., Sukhorukov G.B., (2004):Matrix polyelectrolyte microcapsules: new system for macromolecule encapsulation. Langmuir; 20 (8): 3398-3406.

[30] Zhao Q., Zhang S., Tong W., Gao C.Y., Shen J., (2006): Polyelectrolyte microcapsules templated on poly(styrene sulfonate)-doped $\mathrm{CaCO} 3$ particles for loading and sustained release of daunorubicin and doxorubicin.Eur. Polym. J; $42: 3341$.

[31] Zhao Q. and Li B. (2008): pH-controlled drug loading and release from biodegradable microcapsules. Nanomedicine: Nanotechnology, Biology, and Medicine; 4 : 302-310.

[32] Petrov A. I., Volodkin D. V., Sukhorukov G. B., (2005): Protein-calcium carbonate coprecipitation: A tool for protein encapsulation.Biotechnol. Prog; 21:918-925.

[33] Sui Z. J., Schlenoff J. B., (2004): Phase separations in pH-responsive polyelec-trolyte multilayers: Charge extrusion versus charge expulsion. Langmuir; 20:6026-6031.

[34] Tia Y.Z., Li Y.L., Wang Z., Jiang Y.,(2014): Nuclease-Responsive DNA/PEI Hollow Microcapsules For Bio-Stimuli Controlled Release J . Mater Chem B . Accepted Manuscript. [35] Burke S. E., Barrett C. J., (2003):pH-responsive properties of multilayered poly(Llysine)/hyaluronic acid surfaces.Biomacromol;4: 1773-1783.

[36] Corato R. Di, Palumberi D., Marotta R., Scotto M., Carregal-Romero S., Gil P. R., Parak W. J., Pellegrino T., (2012) : Magnetic nanobeads decorated with silver nanoparticles as cytotoxic agents and photothermal probes .Small; 8: 2731.

[37] De Koker S., De Geest B.G., Singh S.K., De Rycke R., Naessens T., Van Kooyk Y., Demeester J., De Smedt S.C., Grooten J., (2009): Polyelectrolyte microcapsules as antigen delivery vehicles to dendtric cells: uptake, processing, and cross-presentation of encapsulated antigens. Angew. Chem. Int. Ed; 48 : 8485-8489.

[38] Tryoen-Toth P., Vautier D., Haikel Y., Voegel J.C., Schaaf P., Chluba J., Ogier J., (2002): Viability, adhesion, and bone phenotype of osteoblast-like cells on polyelectrolyte multilayer films. J. Biomed. Mater; $60: 657$. 